Published in final edited form as:

Nature. 2009 March 5; 458(7234): 37-38. doi:10.1038/458037a.

\title{
Inside the living cell
}

\author{
David S. Burz and \\ Department of Chemistry, State University of New York at Albany, 1400 Washington Avenue, \\ Albany, New York 12222, USA
}

\section{Alexander Shekhtman}

Department of Chemistry, State University of New York at Albany, 1400 Washington Avenue, Albany, New York 12222, USA

Alexander Shekhtman: ashekhta@albany.edu

\section{Abstract}

Proteins work properly only if they have the correct three-dimensional atomic structure. It is now possible to look at the structures and dynamics of these biological macromolecules as they function inside cells.

The development of structural biology was historically based on the principle of divide and conquer - individual proteins were purified to homogeneity and their atomic structures were solved in vitro by using either X-ray crystallography or nuclear magnetic resonance (NMR) spectroscopy. This approach was tremendously successful, and led to the creation of a protein-structure data-bank that currently contains more than 50,000 structures.

But relating in vitro protein structures to biological processes that occur inside the cell is not a trivial task. A traditional approach to solving this problem entails mutating a protein's structure at certain sites based on its in vitro structure and observing the effects of these changes on the cell. This low-resolution validation of high-resolution structures may still lead to situations where the in vitro structure does not fully represent the physiologically active protein structure under the conditions present in a cell. The work presented by Sakakibara et al. ${ }^{1}$ and Inomata et al. ${ }^{2}$ (pages 102 and 106 of this issue) reveals new ways to solve the structures of proteins as they exist inside living cells, ushering in a fresh era of structural biology.

To determine how protein structures are influenced by their intracellular environment, incell NMR spectroscopy was developed ${ }^{3}$. NMR spectroscopy allows one to directly observe NMR-active isotopes of atomic nuclei within any NMR-inactive environment, and can thus be used to analyse isotopically labelled biomolecules inside unlabelled cells. To date, two approaches have been used to deliver labelled proteins into unlabelled bacterial and animal cells. In the first case, target proteins are produced inside the bacteria by growing them on isotopically labelled media; in the second case, labelled proteins are microinjected into large cells such as Xenopus oocytes (frog eggs) ${ }^{4}$. In these instances, in-cell NMR spectra suggested that protein structures inside cells are very similar to those solved in vitro. The devil, though, is in the detail. 
Changes in protein structure that are caused by specific interactions with well-defined binding partners can be identified by solving in vitro structures of the protein complexes. A more difficult problem is to address how numerous, nonspecific, low-affinity interactions, which are omnipresent in cells, affect a protein's structure. Living cells are extremely crowded environments owing to the high (20-30\% by volume) concentration of macromolecules they contain, and this results in small but potentially important changes in protein structure. The papers published in this issue $e^{1,2}$ make significant steps towards understanding these changes.

A major hurdle to determining in-cell NMR structure is the limited lifetime of the cells inside the NMR sample tube. Standard NMR experiments usually require 1-2 days of data collection, which is an unacceptably long time for live cells. Sakakibara et al. ${ }^{1}$ shortened this time to 2-3 hours by applying a well-known but seldom-used modification of NMR experiments, and thereby determined the three-dimensional structure of a putative heavymetal-binding protein, TTHA1718, expressed inside bacterial cells (Fig. 1a). Their procedure, as described in detail in the paper, may well become a new standard for in-cell NMR.

Comparing the in vitro and in-cell structures of TTHA1718 revealed that, despite marked similarities, there are structural differences, mostly concentrated in the heavy-metal-binding site and in loop regions that undergo dynamic changes as the protein functions. In contrast to the changes in the binding site, which can be explained by metal ions present in the bacterial cytosol, the structural changes seen in the dynamic loop are probably due to molecular crowding and the viscosity of the cytosol that are characteristic of the cell interior. It will be interesting to discover whether this phenomenon is seen when further in-cell NMR structures become available.

Extending in-cell NMR to study proteins inside human cells presents a further challenge. In general, protein production inside these cells does not reach high enough levels for atomicresolution in-cell NMR spectra to be collected. The micro-injection technique is laborious, and up to now has been limited to large cells such as Xenopus oocytes. Inomata et al. describe an innovative method that avoids using microinjection and makes in-cell NMR in human cells possible. By fusing the labelled target protein with a cell-penetrating peptide (Fig. 1b), the target protein can be delivered into the cells, where the peptide tag is then snipped off, allowing the free, labelled target protein to disperse uniformly. Importantly, the authors found that the tag binds to large intracellular structure(s), making it invisible to NMR and thus simplifying the in-cell NMR spectrum of the target protein.

This method opens the door to determining protein structures inside human cells in the near future, but has already been used by Inomata et al. to study in-cell protein dynamics. For example, macromolecular crowding inside the cell should stabilize protein structures. Inomata et al. show that, for at least one protein, ubiquitin, introduced into a human cell at physiological concentrations, the opposite is true. Ubiquitin becomes more dynamic in its reactivity and less structured, presumably due to nonspecific, low-affinity interactions with its binding partners. This unexpected result highlights the importance of studying proteins inside living cells. 
In-cell NMR is limited by the concentration and structural stability of the protein that can be attained inside cells. In addition, some proteins could be difficult to deliver into the cytosol by fusing them with cell-penetrating peptides. Very large proteins, or proteins that bind to large cellular structures, become invisible and cannot be effectively studied by in-cell NMR. The lifetime of the cells is also a limiting factor, because cell breakdown results in protein leaking away into solution, the condition of in vitro NMR. Despite these pitfalls, we are left with an enormous number of proteins that can be studied.

Exploring protein structures and dynamics at the atomic level inside living cells will provide results that cannot be obtained using standard in vitro techniques. The comparative simplicity of the in-cell method allows for a myriad of applications. The regulation of metabolic or signal-transduction pathways, mediated by biomolecular interactions, can now be studied in detail. Drug screening using in-cell NMR can function as an in vivo assay at atomic resolution, providing information about drug delivery inside the cell, where the drug binds, and whether there is a notable difference between how it binds in vivo and in vitro.

More exotic applications include the study of intrinsically unstructured and amyloid-forming proteins in neurons - such proteins having been implicated in neurodegeneration - or of labelled protein probes in diseased tissue. The ability to observe the structures of proteins in their native environment releases the constraints that have previously limited study of protein structure and dynamics to the test tube. Now that structural biology has moved into the cell, it is likely to stay there.

\section{References}

1. Sakakibara D. Nature. 2009; 458:102-105. [PubMed: 19262674]

2. Inomata K, et al. Nature. 2009; 458:106-109. [PubMed: 19262675]

3. Serber Z, et al. J Am Chem Soc. 2001; 123:2446-2447. [PubMed: 11456903]

4. Selenko P, et al. Proc Natl Acad Sci USA. 2006; 103:11904-11909. [PubMed: 16873549]

Nature. Author manuscript; available in PMC 2015 May 21. 
a Bacterial cell

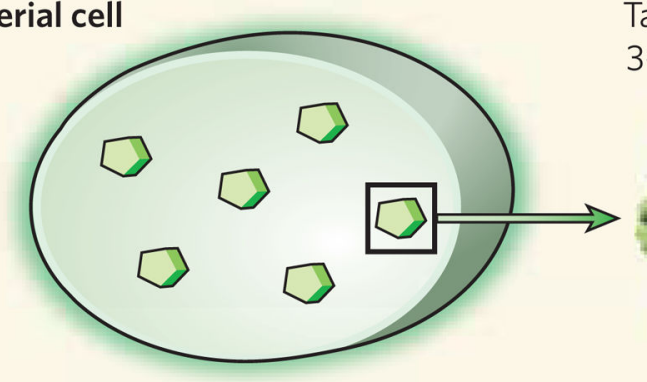

b Human cell

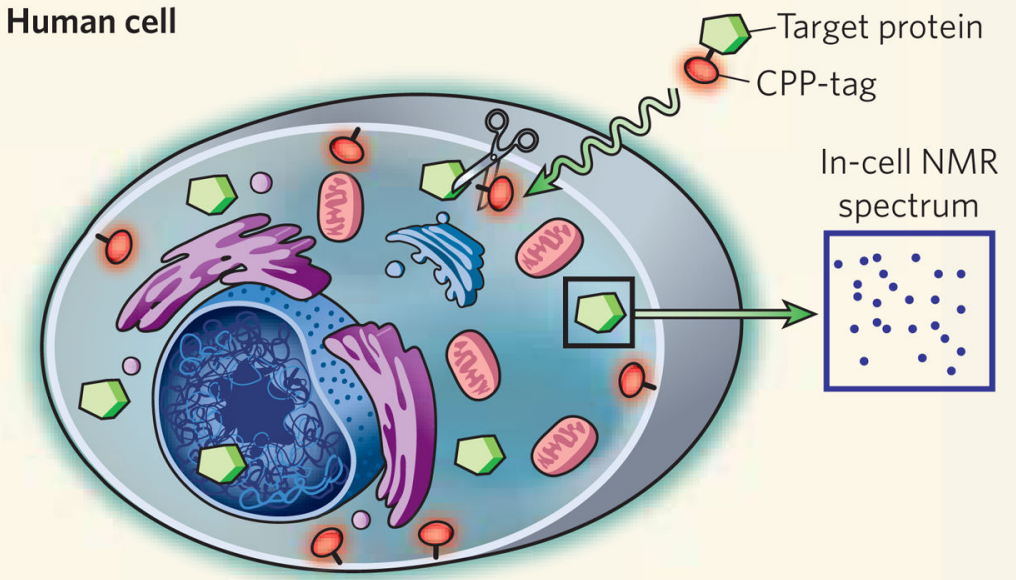

Target protein

3-D structure

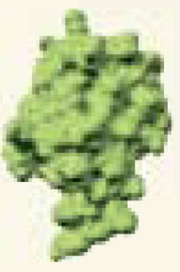

Figure 1. Analysis of proteins using in-cell NMR spectroscopy

a, Sakakibara et al. ${ }^{1}$ show that atomic structures of proteins expressed inside bacterial cells can be solved by in-cell NMR spectroscopy. b. Inomata et al. ${ }^{2}$ fused a target protein with a cell-penetrating peptide (CPP-tag) to transfer stable-isotope-labelled proteins into various human cells and collect in-cell NMR spectra at atomic resolution. This approach has not yet been used to determine protein structures but has been applied by the authors to study protein dynamics. 